\title{
A Study on Incidence and Patterns of Acute Poisoning Cases in an Emergency Department of Western Region of Nepal
}

\author{
Acharya $\mathbf{K}^{1^{*}}$, Kandel IS ${ }^{2}$, Gupta $\mathbf{S}^{3}$, Poudel SD \\ I*Associate Professor, Department of General Practice and Emergency Medicine, GMCTH, ${ }^{2}$ Associate Professor, Department of \\ Orthopedics, GMCTH, ${ }^{3}$ Assistant Professor, Department of General Practice and Emergency Medicine, GMCTH. \\ ${ }^{4}$ Community Health Nursing, TUIOM, Pokhara Nursing Campus, Ramghat Pokhara
}

\section{Keywords}

Emergency,poisoning, organophosphates, Nepal.

\section{Corresponding author}

"Dr. Karuna Acharya, MD General Practice, Associate Professor, Department of General Practice and Emergency Medicine Gandaki Medical College Teaching Hospital, Pokhara, Nepal

Email: karunakandel@gmail.com

\begin{abstract}
Introduction: Poisoning is a common cause of morbidity and mortality in the Nepal. The objective of our study is to evaluate the incidence and pattern of acute poisoning cases admitted to the Gandaki Medical Teaching Hospital.
\end{abstract}

Methods: This is cross-sectional observational study carried out among acute poisoning cases attending emergency department from January 2015 to December 2017.

Results: A total of 156 poisoning cases were treated of which female patients were $71 \%$ and the highest numbers $(36.5 \%)$ of patients were in the age group 21 to 30 years. Organophosphates poisoning was the commonest poison diagnosed among $28.8 \%$, followed by rodenticide poisoning found among 27.5\%. Psychiatric illnesses were present among $94.8 \%$ of our patients. The most common diagnosis were Anxiety and depression $46.6 \%$ followed by family dispute among $30.4 \%$. Above fifty percent of our patients attended our emergency department within 2 hours our ingestion of poisons.

Conclusion: Female and young people are at greater risk of acute poisoning. Pesticides were the most common agent. The reasons for the poisoning need to be carefully evaluated and proper support and advice must be given in poisoning cases.

\section{INTRODUCTION}

Acute poisoning account for significant morbidity and mortality worldwide, especially in developing countries like Nepal. ${ }^{1}$ As per World Health Organization (WHO) estimates 0.3 million people die every year due to various poisoning agents. ${ }^{2}$ Poisoning is one of the major cause of hospitalization through emergency department and is a major public health problem in Nepal. ${ }^{3}$

The nature of use of poisons varies in different parts of the world and may vary even in different parts of the same country depending upon the socio-economic factors and culture diversity. In industrialized countries commonly uses analgesics, tranquillizers and antidepressants agent for mode of poisoning. Whereas developing countries which are agriculture dominant- pesticides agents are the most common methods used. 4,5

Knowledge of general pattern of poisoning in a particular region can help in early diagnosis and treatment, thus contributing to a decrease in mortality and morbidity. Hence the present study was conducted with the main objective to study the incidence and pattern of acute poisoning cases attending Emergency department of 
Gandaki Medical Teaching Hospital, Pokhara, Nepal.

\section{METHODS}

This is cross-sectional observational study carried out among acute poisoning cases attending the Emergency department of Gandaki Medical College Teaching Hospital from January 2015 to December 2017 was enrolled in our study. Approval of this study was obtained from Institutional Review Committee and informed consent was taken from the patients or their family members.

The inclusion criteria included the poisoning cases attending the Emergency Department of Gandaki Medical College Teaching Hospital. The collected data was reviewed verified and statistically analyzed using the Statistics Packed for Social Science (SPSS) version 16 and Microsoft Excel 2007. Descriptive statistics was used for all studied variables.

\section{RESULTS}

One hundred and fifty six patients were included in the study. Our study patient age ranged from two to 69 years. Maximum number of patients-36.5\% belonged in the age group category of 21-30 years followed up by $29.1 \%$ in age group of 11 to 20 years (Figure 1). There were 111 females and 45 males. Maximum above ninety percentage of our patient mode of poisoning was suicidal intention. Almost $60.2 \%$ of our patients were married and $37.1 \%$ of individuals were unmarried. Almost sixty percentages of our patients were from around Pokhara valley area and rest of them was from the rural areas of the districts (Table 1).

Psychiatric illnesses were present among $94.8 \%$ of our patients. The most common diagnosis was Anxiety and depression was $46.6 \%$, family dispute among $30.4 \%$ and mood disorder among $7.4 \%$ of our patients (Table 2). Among the types of poisoning- Organophosphates poisoning was the most common poison diagnosed among $28.8 \%$ of our patients, Rat poisoning was found among $27.5 \%$ and other poisons were found among $16.6 \%$ (Table 3 ). Above fifty seven percent of our patients attended our emergency department within 2 hours our ingestion of poisons followed by twenty eight percent within 2-4 hours and almost fifteen percent after 4 hours of poison ingestion (Table 4).

Age wise Distribution

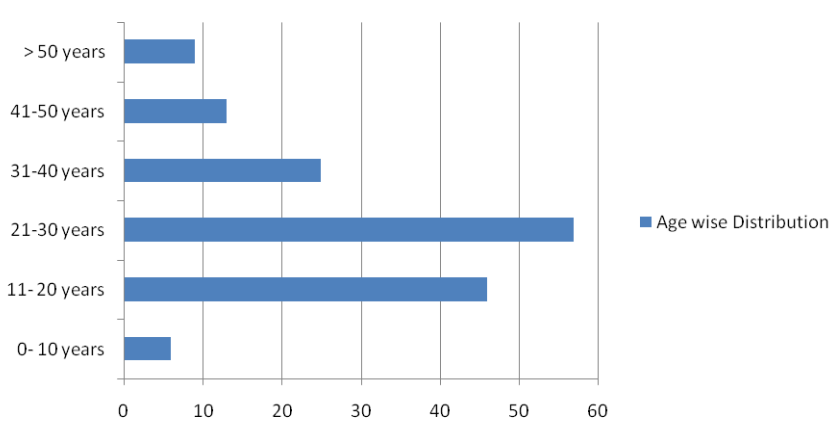

Figure 1: Age wise distribution of patients.

Table 1: Socio demographic distribution of study patients $(\mathrm{N}=156)$

\begin{tabular}{lc}
\hline Patient characteristics & Number (\%) \\
Sex & \\
Male & $45(28.8 \%)$ \\
Female & $111(71.2 \%)$ \\
Mode of poisoning & \\
Suicidal & $148(94.8 \%)$ \\
Accidental & $8(5.2 \%)$ \\
Socioeconomic status & \\
Low & $4(2.6 \%)$ \\
Medium & $152(97.4 \%)$ \\
Urban and rural distribution & \\
Rural & $56(35.9 \%)$ \\
Urban & $100(64.1 \%)$ \\
According to marital status & \\
Married & $94(60.3 \%)$ \\
Unmarried & $58(37.2) \%$ \\
Divorced & $4(2.5 \%)$ \\
\hline
\end{tabular}

Table 2: Psychiatric illness analysis of poisoning patients $(\mathrm{n}=148)$

\begin{tabular}{cc}
\hline Psychiatric Illness & Number (\%) \\
Anxiety and depression & $69(46.6 \%)$ \\
Family dispute & $45(30.4 \%)$ \\
Excess stress and anger & $11(7.4 \%)$ \\
Economic crisis & $10(6.7 \%)$ \\
Fail in exam & $7(4.7 \%)$ \\
Substance abuse & $6(4.2 \%)$ \\
\hline
\end{tabular}


Table 3: According to type of poison $(n=156)$

\begin{tabular}{cc}
\hline Types of Poisoning & Number (\%) \\
Organophosphorous poisoning & $45(28.8 \%)$ \\
Rodenticides & $43(27.6 \%)$ \\
Paracetamol & $6(3.8 \%)$ \\
Mushroom poisoning & $4(2.5 \%)$ \\
Other drugs & $12(7.7 \%)$ \\
Other poison & $26(16.7 \%)$ \\
Unknown & $20(12.9 \%)$ \\
\hline
\end{tabular}

Table 4: Time elapsed since exposure to hospital arrival $(n=156)$

\begin{tabular}{cc}
\hline Hospital Arrival time & Number (\%) \\
Less than 2 hrs & $90(57.6 \%)$ \\
2 - 4 hrs & $45(28.8 \%)$ \\
$>4$ hrs & $21(13.6 \%)$ \\
\hline
\end{tabular}

\section{DISCUSSION}

Poisoning is an increasing common social problem in developing countries like Nepal. Globally, most suicides in low- and middle-income countries are caused by pesticide poisoning. It is estimated that 300000 people die annually in Asia from pesticide ingestion. ${ }^{6}$ This is the first study done on incidence and pattern of poisoning in Emergency department of Gandaki Medical College Teaching Hospital, Pokhara Nepal.

In our study patient age group varied from 2 years to 69 years. Maximum numbers of patients $36.5 \%$ were in age group of 21-30 years. This highlights that adolescents and young adults are the vulnerable age group with increased risk in our community. This finding were similar with study done in Palpa were $24.6 \%$ of patients were in the similar age group. ${ }^{7}$ Suvedi et $\mathrm{al}^{8}$ in a study done at Bir Hospital showed that 14 to 29 years age group were the most age group presenting with poisoning cases in the emergency.

The present study found that the incidence of poisoning were more common in female patients compared to male patients. There were $72 \%$ of female attended our emergency department compared to male $28 \%$ of the total patients. These findings were similar with study done at Manipal teaching hospital, were female patients were $54.08 \%{ }^{9} \mathrm{~A}$ study done in emergency department of Turkey showed female to male ratio of $3.5: 1 .^{10}$ Hence is shows higher prevalence of poisoning cases were reported in female patients compare to males. The reason for the finding may be that females were more emotionally unstable. They are economically dependent on male counterpart. They may suffer from domestic violence and abuse in the family and community as well.

Our study showed above $90 \%$ of poisoning cases was due to suicidal intentional and rest was due to accidentally. In study done at Bir Hospital by Singh et $\mathrm{al}^{3}$ showed $97 \%$ of cases were due to intentional poisoning for suicidal attempt. This shows there is increasing trend of intentional poisoning in the community may be due to undiagnosed psychiatric conditions in the patients like depression and low self esteem. Homicidal poisoning was mainly confined to the effects made by robbers to rob people by giving some fruit juice mixed with sedative agents to drink for the purpose. Demographical pattern showed maximum patient were from around Pokhara valley region as the city is the province capital and people living here for more job opportunities and better education facility.

On evaluation of the co-morbidity health status of our patients showed psychiatric illness analysis commonly present were anxiety and depression $46.6 \%$, family dispute among $30.4 \%$ and mood disorder among $7.4 \%$. There is increase incidence with mental disorder in our patients. Psychiatric evaluation and consultation with support in the community is very important in management of poisoning in Nepal.

Organophosphorus compound was the most common poison found in our study. Almost 30 percent of our patient had taken organophosphorus substance for suicidal intention. These findings were consistent with other studies done in Kafle et $\mathrm{al}^{11}$ and Poudyal et al. ${ }^{12}$ Second in the list was rodenticide containing phosphides substance in $25 \%$. The reason to have increased cases of these substances for poisoning is due to easy and wide-spread availability in our market for agriculture insecticides purposes. In this study, medicinal preparation used for poisoning was $18 \%$. Paracetamol and other drugs available over the counters like analgesics and anxiolytics were more commonly used.

In this study, most of patients $64 \%$ had arrived hospital within two hours after ingestion of poison. In study done by Singh et al almost similar number of patients had arrived hospital within 3 hours. ${ }^{3}$ As our hospital being in center part of Pokhara, we expect patients visiting our hospital. 


\section{CONCLUSIONS}

In developing country like Nepal, poisoning is important public health importance concern hence appropriate intervention program is needed. Female and young people are at greater risk of acute poisoning. Deliberate self harm was the more common reason for poisoning in our subjects. Pesticides were the most common agent. The reasons for the poisoning need to be carefully evaluated and proper support and advice must be given in poisoning cases. Further multi-center study is needed to study the incidence and pattern of poisoning in Nepal.

\section{Acknowledgement}

We would like to express sincere thanks to all the paramedical staffs of Emergency department of Gandaki Medical College and Police of Kaski district for their help in gathering data's and treatment of patients.

\section{REFERENCES}

1. Jeyaratnam J. Acute pesticide poisoning: a major global health problem. World Health Stat. 1990;43:139-44.

2. Thundiyil JG, Stober J, Besbelli N, Pronczuk J. Acute pesticide poisoning: a proposed classification tool. Bull World Health Organ. 2008;86(3):205-9.

3. Singh DP, Aacharya RP. Pattern of poisoning cases in Bir Hospital. Journal of Institute of Medicine. 2006; 28(1):3-6.

4. Karki RK, Risal A. Study of Poisoning Cases in a Tertiary Care Hospital. Kathmandu Univ Med J 2012; 10(4):70-3.

5. McClure GM. Suicide in children and adolescents in England and Wales 1970-1998. Br J Psychiatry. 2001 May; 178:469-74.

6. Gunnell D, Eddleston M, Phillips MR, Konradsen F. The global distribution of fatal pesticide self-poisoning: systematic review. BMC Public Health 2007;7:357.

7. Shakya RP, Adikhari S, Bajracharya R. Pattern of acute poisoning attending a tertiary care hospital of western Nepal. Journal of Lumbini Medical College. 2016;4(2):90-3.

8. Suvedi BK. A retrospective study of poisoning cases at Bir Hospital, Nepal. J Inst Med. 1990;12:296302.
9. Kishore P, Palaian S, Paudel S, Mishra D, Paudel $\mathrm{R}$, Mishra D, et. al. Pattern of poisoning cases in a teaching hospital in Western Nepal. J Inst Med. 2008;30(1):26-34.

10. Gulohglu C, Kara IH. Acute poisoning cases admitted to a university hospital emergency department in Diyarbakir, Turkey. Human and experimental toxicology. 2005;24:49-54.

11. Kafle KK, Gyawali KK. Organ phosphorus commonest poisoning agent. J Inst Med. 1992;14:296-302.

12. Poudyal BP. Poisoning: Pattern and profile of admitted cases in a Hospital in Central Nepal. J Nep Med Assoc. 2002;41:447-56. 